\title{
PRODUTIVIDADE DO TRIGO IRRIGADO SUBMETIDO À APLICAÇÃO DE NITROGÊNIO E À INOCULAÇÃO COM Azospirillum brasilense
}

\author{
Pedro Henrique Marques Paula Nunes(1), Leonardo Angelo Aquino(1)*, Luiz Paulo Dornelas \\ dos Santos(1), Felipe Oliveira Xavier(1), Luciel Rauni Dezordi(1) e Natália Silva Assunção(1) \\ (1) Universidade Federal de Viçosa, Instituto de Ciências Agrárias, Campus de Rio Paranaíba, Rio Paranaíba, Minas Gerais, Brasil. \\ * Autor correspondente. \\ E-mail: leonardo.aquino@ufv.br
}

\begin{abstract}
RESUMO
Inocular sementes de trigo com Azospirillum brasilense pode ser benéfico à cultura do trigo, bem como reduzir a necessidade de aplicação de N. No entanto, são escassos trabalhos que mensurem o benefício dessa prática no trigo irrigado com expectativa de alta produtividade e demanda de $\mathrm{N}$. Objetivou-se com este trabalho avaliar a produtividade e o conteúdo de $\mathrm{N}$ na planta do trigo irrigado, submetido a doses de $\mathrm{N}$, com ou sem a inoculação com A. brasilense. Foram conduzidos dois experimentos em 2011 e repetidos em 2012. Em cada ano de cultivo, instalou-se um experimento na área considerada de baixa disponibilidade de $\mathbf{N}$ no solo (cultivo em sucessão ao milho) e outro na com alta disponibilidade (cultivo em sucessão à soja ou cenoura). Os tratamentos consistiram de cinco doses de $\mathrm{N}$ e da inoculação ou não com A. brasilense. As doses de $\mathrm{N}$ testadas foram 20, 60, 100, $140 \mathrm{e} 180 \mathrm{~kg} \mathrm{ha}^{-1}$ na área com baixa disponibilidade de $\mathrm{N}$ e $20,50,80,110$ e $140 \mathrm{~kg} \mathrm{ha}^{-1}$ na com alta disponibilidade. $\mathrm{O}$ teor de N na folha índice foi aumentado pela inoculação com $A$. brasilense. Entretanto, o $\mathrm{N}$ absorvido não foi influenciado pela inoculação da bactéria. As doses de $\mathrm{N}$ aumentaram a produtividade do trigo e esse aumento foi mais evidente no cultivo na área com baixa disponibilidade de $\mathrm{N}$ no solo.
\end{abstract}

Palavras-chave: Triticum aestivum L., fixação de $\mathrm{N}_{2}$, bactérias diazotróficas. 


\title{
ABSTRACT: YIELD OF THE IRRIGATED WHEAT CROP SUBJECTED TO NITROGEN APPLICATION AND TO INOCULATION WITH Azospirillum brasilense
}

\begin{abstract}
Inoculating wheat seeds with nitrogen-fixing bacteria such as Azospirillum brasilense may be beneficial to the crop and reduce the need for $N$ application. Nevertheless, few studies have measured the benefit of this practice on irrigated wheat with the expectation of high yield and demand for $N$. The objective of this study was to evaluate the yield and $N$ content of irrigated wheat under different $N$ application rates, with and without inoculation with $A$. brasilense. Two experiments were carried out in 2011 and reevaluated in 2012. In each crop year, one experiment was set up in an area with low $N$ availability in the soil (growing in succession to maize) and another with high availability (growing in succession to soybean or carrots). The treatments consisted of five $N$ application rates, and inoculation or no inoculation with A. brasilense. The $N$ rates tested were 20, 60, 100, 140, and $180 \mathrm{~kg}$ ha-1 in the area with low $N$ availability, and 20,50, 80, 110, and $140 \mathrm{~kg} \mathrm{ha}^{-1}$ in the area with high $N$ availability. Yield, grains per spike, dry matter accumulation in the shoots, $N$ content in the index leaf and in the grain, and $N$ uptake and export were evaluated. The $N$ content in the index leaf increased through inoculation with $A$. brasilense, However, the $N$ taken up was not affected by inoculation of the bacteria. The $N$ applied increased wheat yield, and this increase was more evident in the area with low $N$ availability in the soil.
\end{abstract}

Keywords: Triticum aestivum L., $N_{2}$ fixation, diazotrophs.

\section{INTRODUÇÃO}

O trigo (Triticum aestivum L.) é o segundo cereal mais produzido no mundo e em diversos países é a fonte de aproximadamente metade das calorias e proteínas consumidas pela população (Wang et al., 2012). No Brasil, é a cultura de inverno de maior importância, cultivada principalmente nas regiões Sul, Sudeste e Centro-Oeste. No entanto, em razão da grande demanda, ainda depende de importações para suprir o mercado interno (Costa et al., 2013).

O N quando deficiente no solo impõe a maior limitação à produtividade da cultura do trigo (Rodrigues et al., 2014). O adequado suprimento de $\mathrm{N}$ determina o número de perfilhos, o que pode favorecer a formação de nós, e causa maior alongamento do colmo. $\mathrm{O}$ aumento do número de perfilhos e o maior alongamento do colmo permitem maior captação da radiação solar e, com isso, maior produtividade (Fornasieri Filho, 2008). Além disso, o número de espigas por área e o número de espiguetas por espiga aumentam pela adequada disponibilidade e aplicação desse nutriente (Megda et al., 2009).

Em culturas não leguminosas, a adubação nitrogenada representa um dos custos mais elevados do processo produtivo. Os cultivos de trigo, milho e arroz consomem aproximadamente $60 \%$ do total de fertilizantes nitrogenados do mundo (Espíndula et al., 2014). A utilização da fixação biológica do $\mathrm{N}_{2}$ em leguminosas tem-se destacado como alternativa para a redução da aplicação do nutriente, sem comprometer a produtividade dessas. Há grande interesse dos pesquisadores na utilização desse processo biológico para outras culturas, como as gramíneas (Rodrigues et al., 2014).
Os cereais ocupam uma área plantada cinco vezes maior que as leguminosas, podendo haver reduções consideráveis nas quantidades de $\mathrm{N}$ aplicadas, se parte da demanda for suprida pela fixação biológica. Dentre as bactérias capazes de fixar o $\mathrm{N}_{2}$, as do gênero Azospirillum destacam-se nas associações com gramíneas (Radwan et al., 2004).

Além do potencial na fixação do $\mathrm{N}_{2}$, as bactérias do gênero Azospirillum têm sido estudadas quanto à capacidade de promover o crescimento das plantas. Essas bactérias promovem alterações na produção hormonal podendo ocorrer maior crescimento do sistema radicular, o que possibilita maior absorção de água e nutrientes (Bashan e Bashan, 2005). No entanto, em ambiente controlado, observou-se que a inoculação de Azospirillum incrementou a produção de folhas e sem efeito na produtividade de grãos (Rodrigues et al., 2014). São escassas pesquisas para avaliação dos possíveis benefícios da inoculação de Azospirillum no campo, em especial para o trigo irrigado que possui maior potencial produtivo e, por conseguinte, maior demanda por $\mathrm{N}$.

O objetivo deste trabalho foi avaliar a produtividade e o estado nutricional de $\mathrm{N}$ da cultura do trigo irrigado em resposta às doses de $\mathrm{N}$ e à inoculação com Azospirillum brasilense.

\section{MATERIAL E MÉTODOS}

Os experimentos foram conduzidos em 2011 e 2012 na área experimental da Cooperativa Agropecuária do Alto Paranaíba (COOPADAP), situada no município de Rio Paranaíba, MG, $\left(19^{\circ} 12^{\prime} 21^{\prime \prime} \mathrm{S}\right.$ e $46^{\circ} 10^{\prime} 05^{\prime}$ "WO), a $1.140 \mathrm{~m}$ de altitude. 
O clima, segundo a classificação internacional de Köppen, é do tipo Aw, definido como tropical com estação seca. O solo é classificado como Latossolo Vermelho-Amarelo textura argilosa.

Foram conduzidos em cada ano dois experimentos, um em ambiente com baixa disponibilidade de $\mathrm{N}$ no solo e no outro em alta disponibilidade. Considerouse como baixa disponibilidade de $\mathrm{N}$ o cultivo do trigo em sucessão ao milho, que por sua maior relação C:N de modo geral confere menor disponibilidade desse nutriente à cultura em sucessão, em especial nas fases iniciais. Foram consideradas como áreas de alta disponibilidade de $\mathrm{N}$ aquelas em que a cultura antecessora ao trigo foi soja ou cenoura. A soja, por ser leguminosa com associação simbiótica que permite a fixação do $\mathrm{N}_{2}$, e a cenoura, pelas elevadas quantidades de $\mathrm{N}$ aplicadas, deixam elevado resíduo de $\mathrm{N}$ no solo e tendem não ter o efeito de imobilização de $\mathrm{N}$ do solo nas fases iniciais da cultura em sucessão, como é esperado quando o cultivo anterior é o milho. Em ambos os experimentos, foram testadas a presença e ausência de inoculação da bactéria Azospirillum brasilense. $\mathrm{O}$ delineamento experimental utilizado foi de blocos casualizados, em esquema fatorial $5 \times 2$ (cinco doses de $\mathrm{N}$ e com ou sem inoculação com A. brasilense), com quatro repetições.

Em 2011, nos ambientes com baixa e alta disponibilidade de $\mathrm{N}$, o cultivo do trigo sucedeu ao do milho e à soja, respectivamente. Em 2012, sucedeu ao milho e à cenoura, respectivamente. Em cada ambiente (caracterizados anteriormente como baixa ou alta disponibilidade de $\mathrm{N}$ ), os tratamentos constituíram-se de cinco doses de $\mathrm{N}$, com ou sem a inoculação com $A$. brasilense. As doses de $\mathrm{N}$ foram 20,60,100, 140 e $180 \mathrm{~kg} \mathrm{ha}^{-1}$ nos experimentos do ambiente com baixa disponibilidade de $\mathrm{N}$ (sucessão ao cultivo de milho) e de 20,50, 80, 110 e $140 \mathrm{~kg} \mathrm{ha}^{-1}$ de $\mathrm{N}$, naquele com alta disponibilidade (sucessão aos cultivos de soja ou de cenoura). As doses de $\mathrm{N}$ foram estabelecidas considerando a dose de $\mathrm{N}$ recomendada para o trigo irrigado de acordo com Fornasieri Filho (2008), que variam de 80 a $100 \mathrm{~kg} \mathrm{ha}^{-1}$ do nutriente. Foram propostas duas doses menores e duas maiores que essas e com maior amplitude no cultivo em que se esperava maior resposta ao $\mathrm{N}$ (ambiente com baixa disponibilidade de $\mathrm{N}$ no solo, ou seja, cultivo do trigo em sucessão ao milho). Nos tratamentos com inoculação de $A$. brasilense, as sementes foram tratadas com o inoculante Masterfix Gramíneas ${ }^{\circledR}$, na dose de 0,200 L para $100 \mathrm{~kg}$ de semente.

Cada unidade experimental foi constituída por 20 fileiras de cultivo, espaçadas de 0,17 m, com 6,0 m de comprimento. Foram consideradas úteis as 16 fileiras centrais, desprezando-se 1,0 m nas extremidades. Semeou-se, no mês de abril (2011 e 2012), o cultivar BRS 264 para o alcance de 270 plantas $\mathrm{m}^{-2}$.

Amostras de solo nas áreas de cultivo foram caracterizadas quimicamente (Quadro 1). As adubações seguiram as recomendações para a cultura do trigo irrigado em Minas Gerais (Fornasieri Filho, 2008), com exceção da nitrogenada, que teve suas doses preestabelecidas de acordo com os tratamentos.

Em todos os experimentos, o $\mathrm{N}$ foi aplicado na dose de $20 \mathrm{~kg} \mathrm{ha}^{-1}$ na semeadura e o restante de cada dose em cobertura no início do perfilhamento. Optou-se por não parcelar a dose de $20 \mathrm{~kg}$ ha-1 de $\mathrm{N}$ em razão do baixo efeito do parcelamento quando a dose de $\mathrm{N}$ é menor que $60 \mathrm{~kg} \mathrm{ha}^{-1}$ de $\mathrm{N}$ (Espíndula et al., 2014). Em ambas as ocasiões, a fonte de $\mathrm{N}$ aplicada foi a ureia $(44 \%$ de N). Essa foi

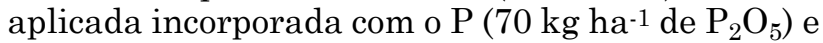
$\mathrm{K}$ (40 kg ha-1 de $\mathrm{K}_{2} \mathrm{O}$ ) na semeadura e distribuída a lanço na superfície do solo na aplicação de cobertura. Imediatamente após a distribuição da ureia em cobertura, foi realizada uma irrigação com lâmina de $15 \mathrm{~mm}$ para incorporação do fertilizante.

O manejo de pragas, doenças e plantas daninhas foi realizado conforme recomendado para a cultura do trigo irrigado (Fornasieri Filho, 2008). O manejo de irrigação foi realizado de acordo com a demanda hídrica da cultura para a região do Alto Paranaíba.

Foram avaliadas as seguintes variáveis: acúmulo de matéria seca da parte aérea, grãos por espiga, massa de 1.000 grãos, produtividade de grãos, teor de $\mathrm{N}$ na folha-índice e nos grãos, $\mathrm{N}$ absorvido e $\mathrm{N}$ exportado.

Para determinar o acúmulo de matéria seca da parte aérea, foram coletadas as plantas de 1,0 $\mathrm{m}$ de

Quadro 1. Caracterização química do solo estudado em 2011 e 2012

\begin{tabular}{|c|c|c|c|c|}
\hline \multirow{2}{*}{ Atributo } & \multicolumn{2}{|c|}{2011} & \multicolumn{2}{|c|}{2012} \\
\hline & BDN & $\mathrm{ADN}$ & BDN & ADN \\
\hline $\mathrm{pH}\left(\mathrm{H}_{2} \mathrm{O}\right)$ & 6,1 & 6,1 & 6,2 & 6,4 \\
\hline $\mathrm{MO}\left(\mathrm{g} \mathrm{kg}^{-1}\right)$ & 34,8 & 45,2 & 38,0 & 36,0 \\
\hline Prem (mg L-1) & 9,2 & 9,2 & 9,2 & 9,2 \\
\hline $\mathrm{P}\left(\mathrm{mg} \mathrm{dm}{ }^{-3}\right)$ & 20,4 & 16,8 & 49,2 & 32,4 \\
\hline $\mathrm{K}(\mathrm{mg} \mathrm{dm}-3)$ & 97 & 100 & 125 & 129 \\
\hline $\mathrm{Ca}^{2+}\left(\mathrm{cmol}_{\mathrm{c}} \mathrm{dm}^{-3}\right)$ & 3,15 & 3,49 & 3,40 & 3,60 \\
\hline $\mathrm{Mg}^{2+}\left(\mathrm{cmol}_{\mathrm{c}} \mathrm{dm}^{-3}\right)$ & 0,72 & 0,58 & 1,2 & 1,9 \\
\hline $\mathrm{SB}\left(\mathrm{cmol}_{\mathrm{c}} \mathrm{dm}^{-3}\right)$ & 4,12 & 4,33 & 4,92 & 5,83 \\
\hline $\mathrm{CTC}_{\mathrm{pH} 7}\left(\mathrm{cmol}_{\mathrm{c}} \mathrm{dm}^{-3}\right)$ & 7,94 & 7,93 & 7,29 & 9,04 \\
\hline V (\%) & 51,9 & 54,6 & 67,5 & 64,5 \\
\hline $\mathrm{B}\left(\mathrm{mg} \mathrm{dm} \mathrm{m}^{-3}\right)$ & 0,51 & 0,62 & 0,84 & 0,71 \\
\hline $\mathrm{Zn}\left(\mathrm{mg} \mathrm{dm}^{-3}\right)$ & 18,8 & 2,3 & 8,9 & 7,4 \\
\hline $\mathrm{Fe}(\mathrm{mg} \mathrm{dm}-3)$ & 44,0 & 46,2 & 59,0 & 49,0 \\
\hline $\operatorname{Mn}(\mathrm{mg} \mathrm{dm}-3)$ & 28,8 & 9,4 & 3,7 & 2,3 \\
\hline $\mathrm{Cu}\left(\mathrm{mg} \mathrm{dm}^{-3}\right)$ & 8,0 & 1,1 & 4,1 & 2,2 \\
\hline
\end{tabular}

MO: matéria orgânica - método de óxido redução; P, K, Zn, Fe, $\mathrm{Mn}$ e Cu: extrator Mehlich-1; B: água quente; $\mathrm{Ca}^{2+}, \mathrm{Mg}^{2+}$ : extrator $\mathrm{KCl} 1 \mathrm{~mol} \mathrm{~L}^{-1}$; SB: soma de bases; $\mathrm{CTC}_{\mathrm{pH}}$ : capacidade de troca de cátions; BDN: área com baixa disponibilidade de $\mathrm{N}$ no solo; e ADN: área com alta disponibilidade de $\mathrm{N}$ no solo. 
fileira, que foram cortadas rente ao solo, no estádio de grãos pastosos. As plantas foram secas em estufa com ventilação forçada de ar a $70^{\circ} \mathrm{C}$ por $72 \mathrm{~h}$.

No início do florescimento, realizou-se a coleta de 50 folhas-índice por unidade experimental. Essa folha corresponde àquela completamente expandida abaixo da espiga por ocasião do florescimento (Argenta et al., 2001). As amostras de folha-índice foram secas em estufa com ventilação forçada de ar a $70^{\circ} \mathrm{C}$ por $72 \mathrm{~h}$ e trituradas em moinho tipo Willey. A quantificação do teor de $\mathrm{N}$ seguiu o método descrito por Malavolta et al. (1997). A partir das informações de teor foliar de $\mathrm{N}$ e matéria seca, calcularam-se as quantidades de $\mathrm{N}$ absorvidas ( $\mathrm{N}$ acumulado na parte vegetativa + grãos) e de $\mathrm{N}$ exportado ( $\mathrm{N}$ acumulados nos grãos).

A colheita da cultura foi realizada 120 dias após a semeadura. Foram tomadas 10 espigas por unidade experimental, para contagem de grãos por espiga. Foram colhidas plantas de $12 \mathrm{~m}$ de fileiras centrais para determinar a produtividade. As plantas foram submetidas à trilha, os grãos pesados e a produtividade calculada com umidade corrigida para $14,9 \%$ na base seca. Uma amostra dos grãos colhidos foi tomada para definir a massa de 1.000 grãos secos.

Os dados foram submetidos à análise de variância conjunta dos dois anos de avaliação. Os graus de liberdade dos fatores estudados foram desdobrados. Quando houve efeito significativo de pelo menos um fator em estudo, procedeu-se à análise de regressão para as doses de $\mathrm{N}$ para cada ano de cultivo, com ou sem a inoculação de $A$. brasilense, e para cultivos com baixo ou alto residual de $\mathrm{N}$ no solo.

\section{RESULTADOS E DISCUSSÃO}

A matéria seca da parte aérea (MSPA) não foi influenciada pela inoculação de $A$. brasilense independentemente da disponibilidade de $\mathrm{N}$ no solo, exceto em 2011 no solo com baixa disponibilidade de N. Nesse ano, a inoculação com $A$. brasilense (média das doses de N) reduziu a MSPA (Quadro 2).

Quadro 2. Efeito da inoculação com Azospirillum brasilense sobre a matéria seca da parte aérea (MSPA), grãos por espiga (GRE), massa de 1.000 grãos (MMG), produtividade (PROD), teor de $\mathrm{N}$ na folha-índice (NFI), teor de N nos grãos (NGR), N absorvido (NA) e N exportado pelos grãos (NE) do trigo cultivado em solo com baixa ou alta disponibilidade de $\mathrm{N}$ no solo, em 2011 e 2012

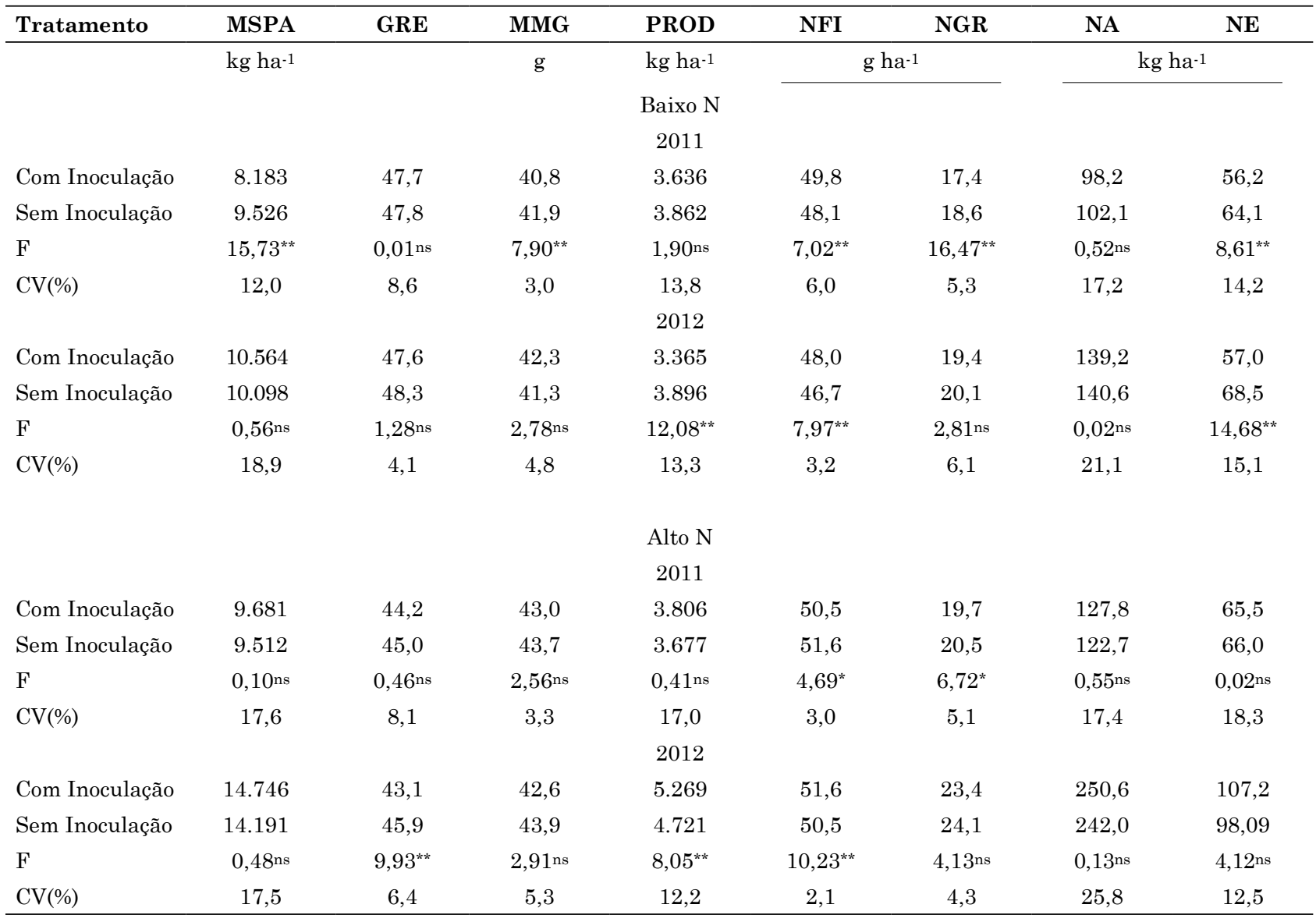

$\mathrm{CV}$ : coeficiente de variação; ${ }^{* *}, \mathrm{e}^{\text {ns }}$ significativos a 1 e $5 \%$, e não significativo pelo teste $\mathrm{F}$, respectivamente. 
No entanto, a MSPA aumentou linearmente com as doses de $\mathrm{N}$, independentemente da disponibilidade de N no solo, em 2011 (Figura 1a,b). Em 2012, a MSPA foi influenciada pelas doses de $\mathrm{N}$, apenas no cultivo em solo com baixa disponibilidade de N (Figura 1a,b).

Geralmente, cultivos de leguminosas e hortaliças deixam residual considerável de $\mathrm{N}$ no solo, seja por meio dos resíduos culturais ou pela adubação com o nutriente (Prando et al., 2012). Isso explica a ausência de resposta à adubação nitrogenada sobre a MSPA no cultivo em solo com alta disponibilidade de $\mathrm{N}$ em 2012, cujo cultivo anterior foi de cenoura. Sala et al. (2007) e Lana et al. (2012) verificaram incremento na matéria seca do trigo, com a inoculação com Azospirillum, diferentemente do observado neste trabalho. Diferenças no residual de $\mathrm{N}$ nos solos utilizados por esses autores e nos utilizados nesta pesquisa podem explicar em parte o não efeito da inoculação. Ainda, bactérias do gênero Azospirillum são nativas do solo (Sala et al., 2007). É possível que essas se encontrassem em alta população nos solos em estudo e, com isso, anulasse efeito da inoculação.

O número de grãos por espiga (GRE) não foi influenciado pela inoculação de $A$. brasilense, em solo com baixa disponibilidade de N, em ambos os anos; e em 2011, na área com alta disponibilidade de N, resultado semelhante ao encontrado por Rodrigues et al. (2014). Em contrapartida, no solo com alta disponibilidade de N, em 2012, houve redução do número de grãos por espiga com a inoculação da bactéria. $\mathrm{Na}$ ausência da inoculação, obteve-se média de 46 grãos por espiga, enquanto na presença da bactéria as espigas apresentaram média de 43 grãos. Independentemente da disponibilidade de $\mathrm{N}$, a dose desse nutriente não influenciou o GRE em ambos os anos de cultivo (Figura 2a,b).

Em 2011, em solo com baixa disponibilidade de N, a massa de 1.000 grãos (MMG) foi menor nos tratamentos em que se realizou a inoculação. Em 2012, essa variável não foi influenciada pela inoculação (Quadro 2). As doses de $\mathrm{N}$ não alteraram a MMG em ambos os anos de cultivo, na área com alta disponibilidade de $\mathrm{N}$ no solo (Figura 2d). Naquela com baixa disponibilidade de N, em 2012, a MMG apresentou decréscimo linear com o aumento das doses de N (Figura 2c). Isso pode ter ocorrido por causa da maior partição de fotoassimilados para o acúmulo de biomassa vegetativa, em detrimento ao enchimento de grãos. Resultados semelhantes aos deste trabalho foram encontrados por Rodrigues et al. (2014), os quais verificaram redução na MMG quando inoculado com A. brasilense (Quadro 2), e diferentes dos obtidos por Didonet et al. (2000), os quais notaram que a MMG foi maior em plantas inoculadas comparadas as não inoculadas. Os autores afirmaram que a inoculação de $A$. brasilense melhorou a distribuição de fotoassimilados na planta em benefício da produção de grãos. (a)

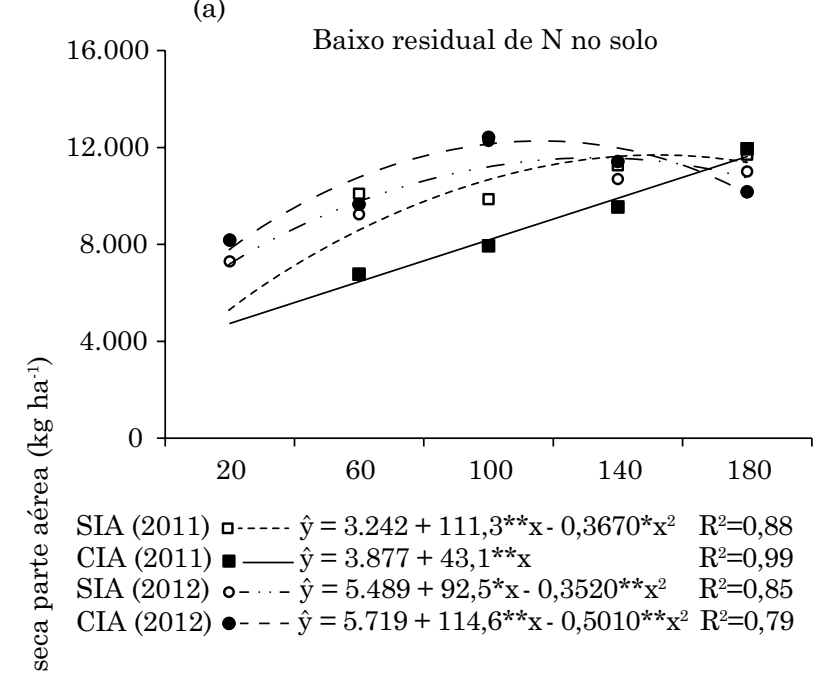

(b)

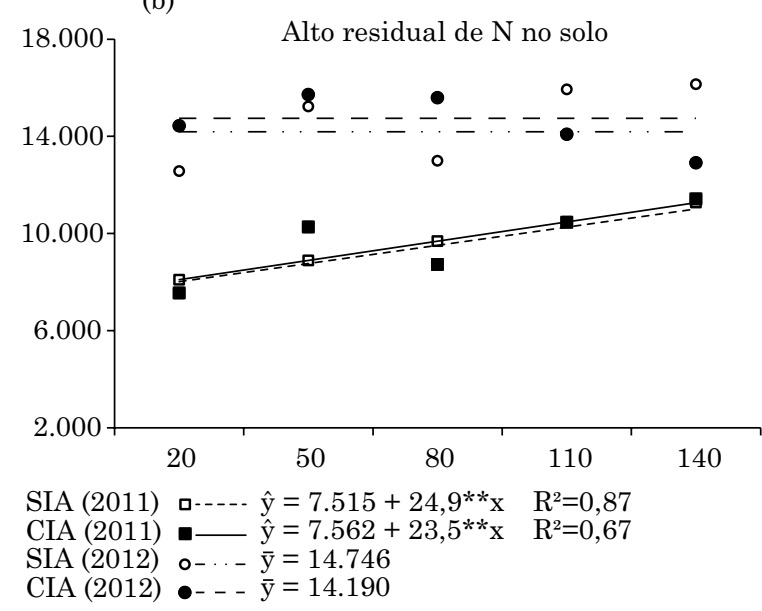

Dose de N $\left(\mathrm{kg} \mathrm{ha}^{-1}\right)$

Figura 1. Matéria seca da parte aérea do trigo em função de doses de nitrogênio e da inoculação ou não com Azospirillum brasilense. SIA: sem inoculação com $A$. brasilense; e CIA: inoculação com $A$. brasilense. ${ }^{* *} \mathrm{e}$ *: significativos a 1 e $5 \%$, respectivamente, pelo teste $t$.

Em 2011, a produtividade de grãos não foi influenciada pela inoculação de $A$. brasilense em ambas as áreas (Quadro 2). Em 2012, a inoculação aumentou em $10 \%$ a produtividade do trigo no ambiente com alta disponibilidade de $\mathrm{N}$ quando comparada à não inoculação (5.269 e $4.721 \mathrm{~kg} \mathrm{ha}^{-1}$, respectivamente). Nesse mesmo ano, no ambiente com baixa disponibilidade de $\mathrm{N}$, a produtividade foi $15 \%$ menor (3.365 e $3.896 \mathrm{~kg} \mathrm{ha}^{-1}$, respectivamente), quando as sementes foram inoculadas com $A$. brasilense. Algumas cultivares de trigo apresentam aumento da produção de grãos com a inoculação com $A$. brasilense, podendo alcançar incrementos da ordem de 27 a $45 \%$ em relação ao controle (Sala et al., 2007). 

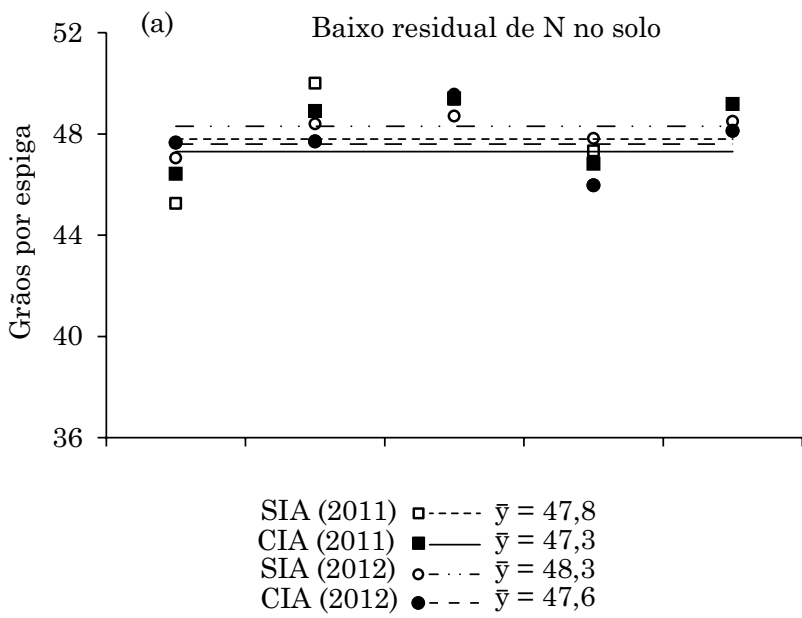

(c)

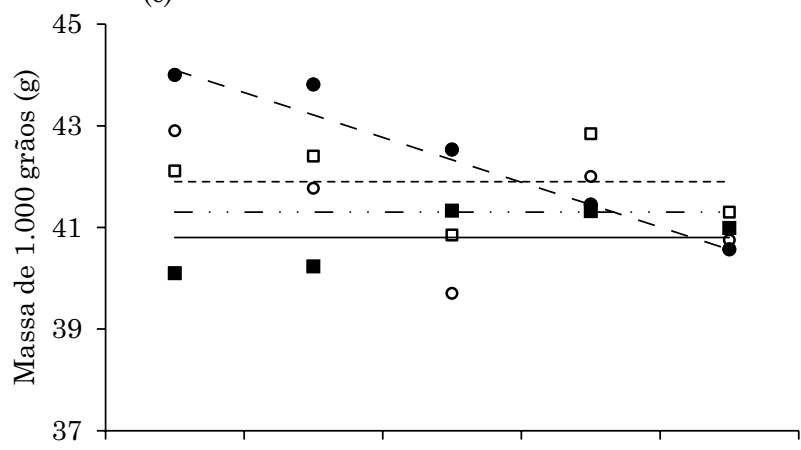

SIA (2011)

CIA (2011) - $\overline{\mathrm{y}}=43,0$

SIA (2012) o- ... - $\bar{y}=43,9$

CIA (2012) - - - $\hat{\mathrm{y}}=44,54-0,022^{*} \mathrm{x} \mathrm{R}^{2}=0,69$

(e)

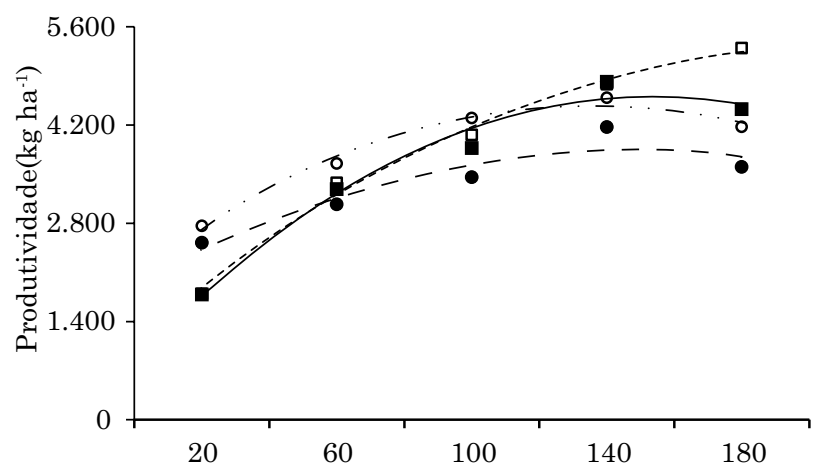

SIA (2011) CIA (2011) - - $\hat{y}=894+47,6^{* *} x-0,1530^{* *} x^{2} \quad R^{2}=0,97$ SIA (2012) $-\cdots-\hat{\mathrm{y}}=2.053+35,9^{* *} \mathrm{x}-0,1320^{* *} \mathrm{x}^{2} \mathrm{R}^{2}=0,99$ CIA (2012) - - - $\hat{y}=1.973+25,1^{* *} \mathrm{x}-0,0850 * \mathrm{x}^{2} \quad \mathrm{R}^{2}=0,88$ (b) Alto residual de $\mathrm{N}$ no solo

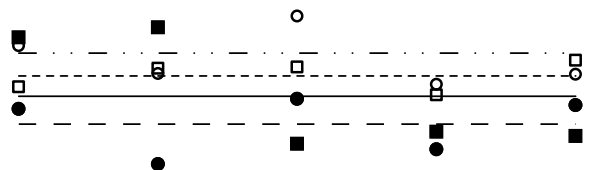

SIA (2011) $\square-\cdots \bar{y}=45,0$
CIA (2011) - - $-\bar{y}=44,2$
SIA (2012) $\bullet-\cdots-\bar{y}=45,9$
CIA (2012) $\bullet---\bar{y}=43,1$

(d)

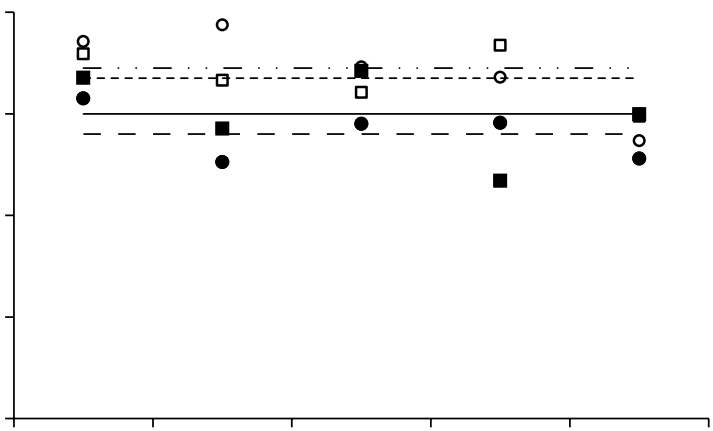

SIA (2011)

CIA (2011) - $\quad \bar{y}=43,0$

SIA (2012) $0-\cdots-\bar{y}=43,9$

CIA (2012) - - - $\bar{y}=42,6$

(f)

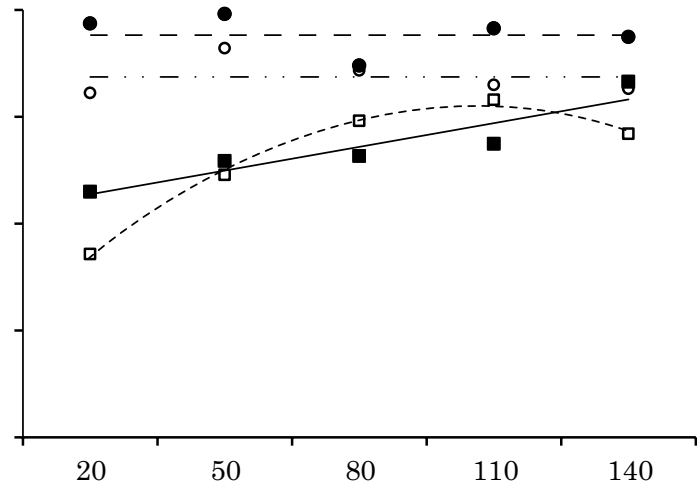

SIA (2011) $\square-\cdots \hat{y}=1.333+56,9^{* *} \mathrm{x}-0,26949^{* *} \mathrm{x}^{2} \quad \mathrm{R}^{2}=0,99$ CIA (2011) - - $\hat{\mathrm{y}}=2.977+10,4^{* *} \mathrm{x} \quad \mathrm{R}^{2}=0,86$ SIA (2012) o- - - $\bar{y}=4.721$

CIA $(2012) \bullet---\bar{y}=5.269$

Dose de N (kg ha $\left.{ }^{-1}\right)$

Figura 2. Número de grãos por espiga, massa de 1.000 grãos e produtividade do trigo em função de doses de N e da inoculação ou não com Azospirillum brasilense. ** e * significativos a 1 e $5 \%$, respectivamente, pelo teste t. SIA: sem inoculação com Azospirillum brasilense; e CIA: com inoculação de A. brasilense. 

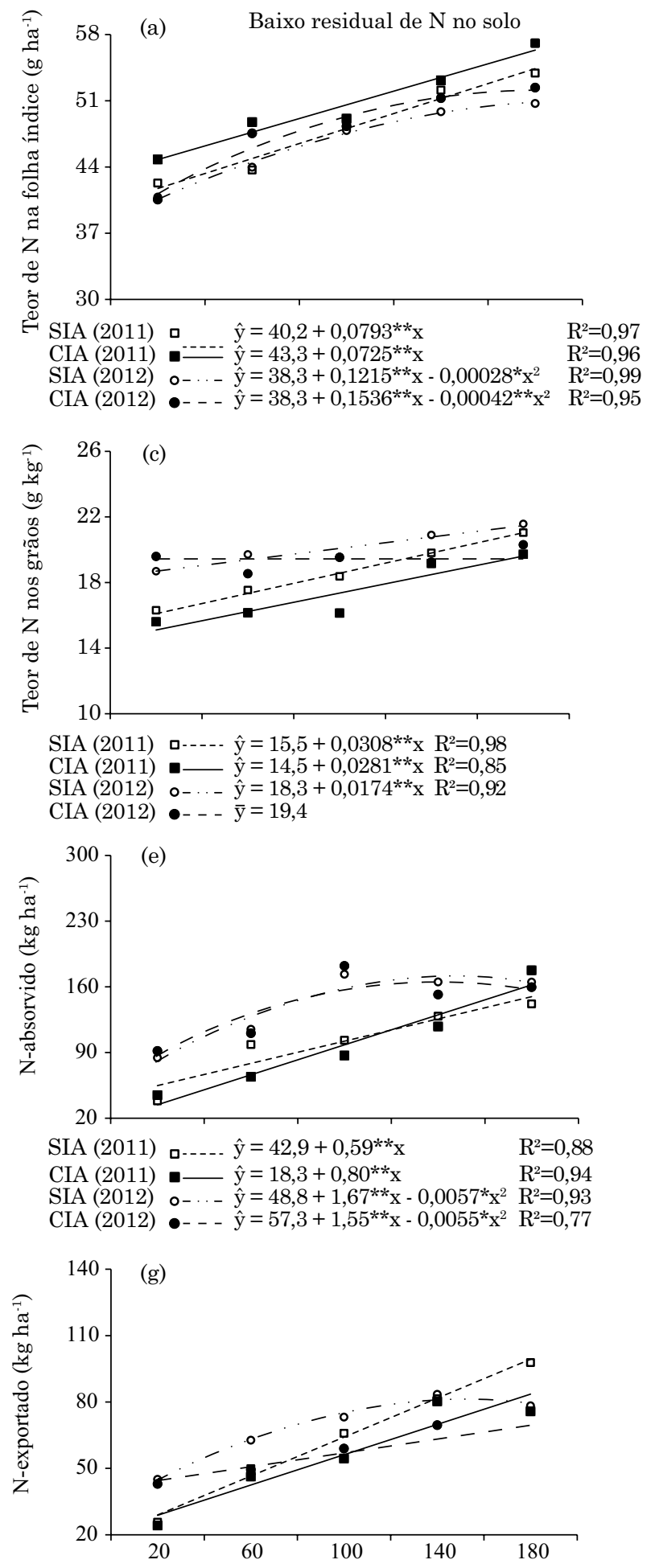

SIA (2011) -.-.-. $\hat{y}=40,2+0,079 * * x \quad R^{2}=0,97$ CIA (2011) - - $\hat{\mathrm{y}}=21,9+0,343^{* *} \mathrm{x} \quad \mathrm{R}^{2}=0,90$ SIA (2012) $-\cdots-\hat{y}=32,8+0,628^{* *} \mathrm{x}-0,0021^{*} \mathrm{x}^{2} \quad \mathrm{R}^{2}=0,98$ CIA (2012) • - - - $\hat{y}=41,4+0,156^{* *} \mathrm{x} \quad \mathrm{R}^{2}=0,84$
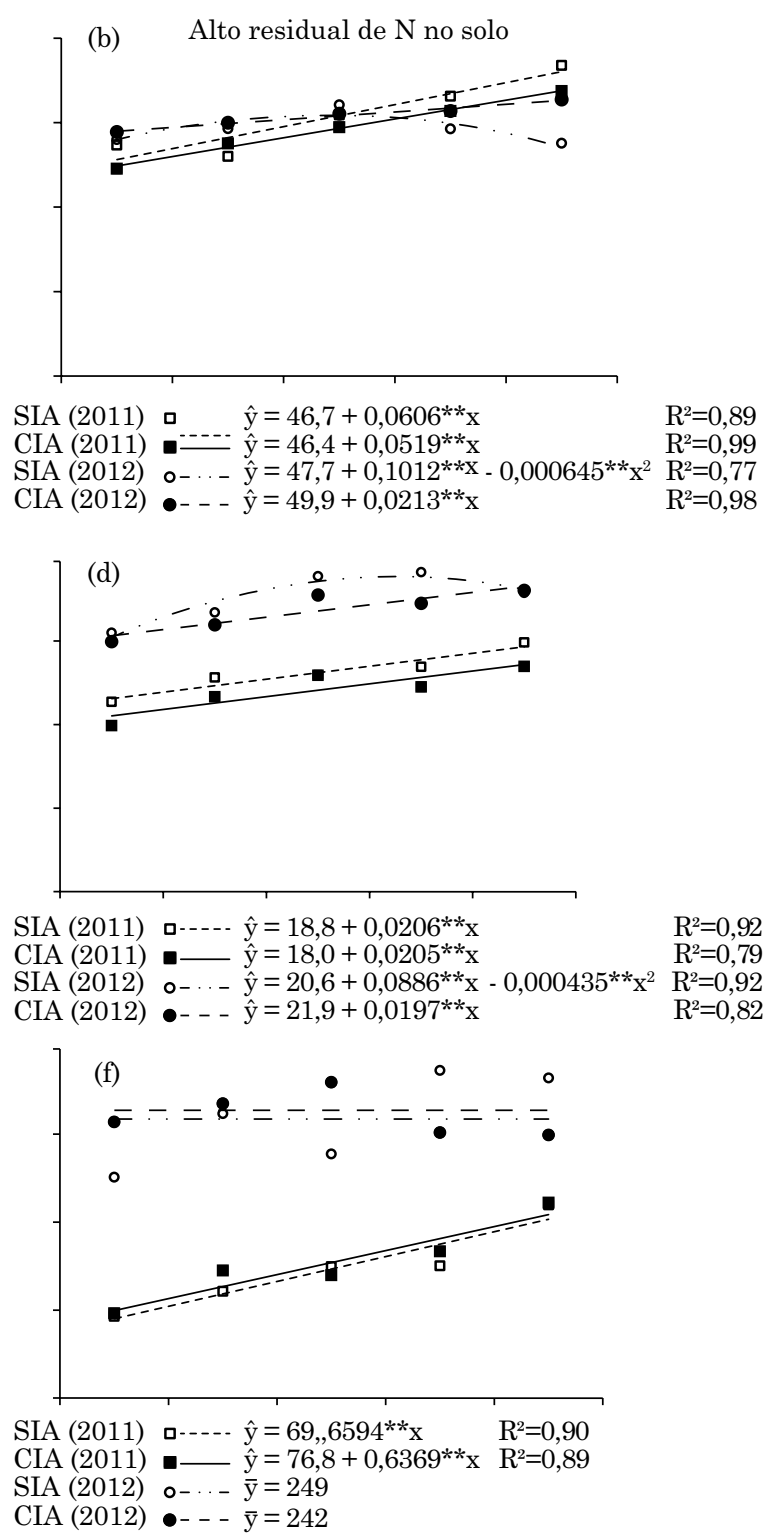

(h)

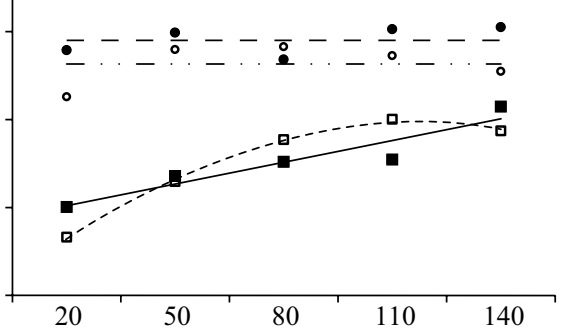

SIA (2011) $\square-\cdots \hat{y}=21,4+1,0065^{* * *} \mathrm{x}-0,00437^{*} \mathrm{x}^{2} \mathrm{R}^{2}=0,99$ CIA (2011) - - $\hat{\mathrm{y}}=45,7+0,2468^{* *} \mathrm{x} \quad \mathrm{R}^{2}=0,89$

SIA (2012) $0-\cdots-\bar{y}=107$

CIA (2012) $\bullet-\bar{y}=99$
$\mathrm{R}^{2}=0,92$ $\mathrm{R}^{2}=0,79$ $\mathrm{R}^{2}=0,92$ $\mathrm{R}^{2}=0,82$

Dose de $\mathrm{N}\left(\mathrm{kg} \mathrm{ha}^{-1}\right)$

Figura 3. Teor de $\mathrm{N}$ na folha-índice, teor de $\mathrm{N}$ nos grãos, $\mathrm{N}$ absorvido e $\mathrm{N}$ exportado pelo trigo em função de doses de $\mathrm{N}$ e da inoculação ou não com Azospirillum brasilense. ** e * significativos a 1 e $5 \%$, respectivamente, pelo teste t. SIA: sem inoculação com Azospirillum brasilense; e CIA: com inoculação de $A$. brasilense. 
A produtividade de grãos foi incrementada com as doses de $\mathrm{N}$, com exceção do solo com alta disponibilidade, em 2012 (Figura 2e,f). A ausência de resposta às doses de $\mathrm{N}$ nessa condição de cultivo pode ser explicada pela maior disponibilidade de $\mathrm{N}$ no solo, visto que na cultura antecessora (cenoura) utilizaram altas doses desse nutriente. Assim, o solo pode suprir $\mathrm{N}$ para o trigo em quantidade suficiente independentemente da aplicação de $\mathrm{N}$.

Prando et al. (2012) obtiveram em solo com alto resíduo de $\mathrm{N}$ (sucessão ao cultivo de leguminosas) produtividade de grãos de $4.347 \mathrm{~kg} \mathrm{ha}^{-1}$, semelhante à alcançada neste trabalho. Prando et al. (2013) relataram que é possível, em solo com elevado residual de $\mathrm{N}$, obtenção de produtividades acima de $3.500 \mathrm{~kg} \mathrm{ha}^{-1}$ somente com adubação de plantio. $\mathrm{O}$ bom suprimento de $\mathrm{N}$ do solo favorece o maior número de perfilhos e grãos por espiga, que influenciam os componentes de produtividade: o número de espigas por área e de grãos por espiga (Megda et al., 2009).

A inoculação com $A$. brasilense aumentou o teor de $\mathrm{N}$ na folha-índice (NFI) em ambos os anos e ambientes de cultivo, exceto em alta disponibilidade de $\mathrm{N}$ no solo em 2011 em que houve decréscimo significativo com a inoculação (Quadro 2). O NFI aumentou com as doses de $\mathrm{N}$ em todos os experimentos (Figura 3a,b). Os teores de $\mathrm{N}$ foram superiores aos da faixa considerada adequada por Pauletti (2004) para a cultura do trigo e aos obtidos por Teixeira Filho et al. (2010).

$\mathrm{O}$ teor de $\mathrm{N}$ nos grãos (NGR) foi maior na ausência da inoculação em ambas as áreas de cultivo, em 2011. Em 2012, não houve influência da inoculação sobre o NGR (Quadro 2). Sala et al. (2007) não verificaram diferença nos teores de $\mathrm{N}$ nos grãos em resposta à inoculação com a bactéria. Porém, Rampim et al. (2012) e Souza et al. (2014) verificaram que a inoculação com Azospirillum incrementou o teor de N nos grãos. Rampim et al. (2012) ainda relataram que a inoculação promoveu alterações na morfologia das raízes e no crescimento das plantas. O NGR aumentou com as doses de N, em ambos os anos e ambientes, com exceção da área de baixa disponibilidade de $\mathrm{N}$ com inoculação de $A$. brasilense, em 2012 (Figura 3c,d). Prando et al. (2012) e Souza et al. (2014) também notaram aumento linear no teor de $\mathrm{N}$ nos grãos com o incremento de suas doses. $\mathrm{O}$ aumento de $\mathrm{N}$ nos grãos é interessante, dada sua associação com o aumento no teor de proteínas e, por conseguinte, na qualidade tecnológica da farinha de trigo (Cazetta et al., 2008).

O N-absorvido (NA) - conteúdo de $\mathrm{N}$ na parte aérea do trigo - não foi influenciado pela inoculação de $A$. brasilense em ambos os ambientes e anos de cultivos (Quadro 2). O acúmulo de $\mathrm{N}$ na parte aérea aumentou com as doses de $\mathrm{N}$, em especial no cultivo em ambiente com menor disponibilidade de $\mathrm{N}$ (Figura 3e,f). Nesse ambiente, a dose de $147 \mathrm{~kg}^{\mathrm{h}} \mathrm{A}^{-1}$ de $\mathrm{N}$ proporcionou o maior acúmulo de $\mathrm{N}$ na planta (171 kg ha-1). No solo com alta disponibilidade de N, as plantas de trigo acumularam $249 \mathrm{~kg} \mathrm{ha}^{-1}$ de N. Esse acúmulo é maior que as doses de $\mathrm{N}$ utilizadas na pesquisa, o que indica que o resíduo de $\mathrm{N}$ do solo pode ser significativo para atender à demanda da cultura quanto a esse nutriente. Sala et al. (2007), em estudo com adubação nitrogenada em trigo, encontraram acúmulo máximo de $193 \mathrm{~kg} \mathrm{ha}^{-1}$ de N, valor semelhante aos encontrados nesta pesquisa.

$\mathrm{O} \mathrm{N}$-exportado (NE) - N acumulado nos grãos - não foi influenciado pela inoculação com A. brasilense em ambos os anos, quando cultivado sobre alta disponibilidade de $\mathrm{N}$ no solo. No cultivo em baixa disponibilidade de N, o NE diminuiu com a inoculação nos dois anos (Quadro 2). O NE aumentou com as doses de $\mathrm{N}$ exceto em 2012 no cultivo com alta disponibilidade de N (Figura 3g,h). Prando et al. (2012) relataram valores maiores que os encontrados neste trabalho para o NE. Tais diferenças podem ser atribuídas aos cultivares e ao ambiente de cultivo.

\section{CONCLUSÕES}

A produtividade do trigo aumentou com as doses de $\mathrm{N}$ e, de forma mais expressiva, no cultivo em sucessão ao milho.

A inoculação com $A$. brasilense não influenciou positivamente as variáveis de crescimento, produtividade e acúmulo de $\mathrm{N}$ no trigo.

Os resultados com a inoculação de $A$. brasilense nem sempre seguiram a mesma tendência, podendo aumentar ou reduzir determinada variável, o que sugere a necessidade de mais estudos a respeito do assunto.

\section{AGRADECIMENTOS}

À FUNARBE - Fundação Arthur Bernardes, pelo auxílio financeiro à pesquisa; ao $\mathrm{CNPq}$, pela concessão de bolsas IC ao primeiro e terceiro autores; e à FAPEMIG, pela bolsa IC concedida ao quarto autor.

\section{REFERÊNCIAS}

Argenta G, Silva PRF, Bortolini CG. Clorofila na folha como indicador do nível de nitrogênio em cereais. Ci Rural. 2001;31:715-22.

Bashan Y, Bashan LE. Plant growth promoting. In: Hillel D, editor. Encyclopedia of soil in the environment. Oxford: Elsevier; 2005. p.103-15. 
Cazetta DA, Asieri Filho D, Arf O, Germani R. Qualidade industrial de cultivares de trigo e triticale submetidos à adubação nitrogenada no sistema de plantio direto. Bragantia. 2008;67:741-50.

Costa L, Zucareli C, Riede CR. Parcelamento da adubação nitrogenada no desempenho produtivo de genótipo de trigo. $\mathrm{R}$ Ci Agron. 2013;44:215-24.

Didonet AD, Lima OS, Candaten MH, Rodrigues O. Realocação de nitrogênio e de biomassa para os grãos, em trigo submetido a inoculação de Azospirillum. Pesq Agropec Bras. 2000;35:401-11.

Espíndula MC, Rocha VS, Souza MA, Campanharo M, Pimentel AJB. Urease inhibitor (NBPT) and efficiency of single or Split application of urea in wheat crop. R Ceres. 2014;61:273-79.

Fornasieri Filho D. Manual da cultura do trigo. Jaboticabal: Fundação de Apoio à Pesquisa, Ensino e Extensão; 2008.

Lana MC, Dartora J, Marini D, Hann JE. Inoculation with Azopirillum, associated with nitrogen fertilization in maize. $\mathrm{R}$ Ceres. 2012;59:399-405.

Malavolta E, Vitti GC, Oliveira SA. Avaliação do estado nutricional das plantas. Princípios e aplicações. $2^{\text {a }}$ ed. Piracicaba: Associação Brasileira para Pesquisa da Potassa e do Fosfato; 1997.

Megda MM, Buzetti S,Andreotti M, Teixeira Filho MMC, Vieira MX. Resposta de cultivares de trigo ao nitrogênio em relação às fontes e épocas de aplicação sob plantio direto e irrigação por aspersão. Ci Agrotec. 2009;33:1055-60.

Pauletti V. Nutrientes: Teores e interpretações. Castro: Fundação ABC; 2004.

Prando AM, Zucareli C, Fronza V, Bassoi MC, Oliveira FA. Formas de uréia e doses de nitrogênio em cobertura no desempenho agronômico de genótipos de trigo. Semina: Ci Agrár. 2012;33:621-32.
Prando AM, Zucareli C, Fronza V, Oliveira FA, Oliveira Júnior A. Características produtivas do trigo em função de fontes e doses de nitrogênio. Pesq Agropec Trop. 2013;43:34-41.

Radwan TEE, Mohamed ZK, Reis VM. Efeito da inoculação de Azospirillum e Herbaspirillum na produção de compostos indólicos em plântulas de milho e arroz. Pesq Agropec Bras. 2004;39:987-94.

Rampim L, Rodrigues-Costa ACP, Nacke H, Klein J, Guimarães VF. Qualidade fisiológicas de sementes de três cultivares de trigo submetidas à inoculação e diferentes tratamentos. R Bras. Sementes. 2012;34:678-85.

Rodrigues LFOS, Guimarães VF, Silva MB, Pinto Junior AS, Klein J, Costa ACPR. Características agronômicas do trigo em função de Azospirillum brasilense, ácidos húmicos e nitrogênio em casa de vegetação. R Bras Eng Agríc Amb. 2014;18:31-7.

Sala VMR, Cardoso EJBN, Freitas JG, Silveira APD. Resposta de genótipos de trigo à inoculação de bactérias diazotróficas em condições de campo. Pesq Agropec Bras. 2007;42:833-42.

Souza TM, Prando AM, Takabayashi CR, Santos JS, Ishikawa AT, Felício ALSM, et al. Composição química e desoxinivalenol em trigo da região Centro - Sul do Paraná: Adubação nitrogenada em cobertura associada com Azospirillum brasilense. Semina: Ci Agrár. 2014;35:327-42.

Teixeira Filho MCM, Buzetti S, Andreotti M, Arf O, Benett CGS. Doses, fontes e épocas de aplicação de nitrogênio em trigo irrigado em plantio direto. Pesq Agropec Bras. 2010;45:797-804.

Wang J, Mao H, Zhao H, Huang D, Wang Z. Different increases in maize and wheat grain zinc concentrations caused by soil and foliar applications of zinc in Loess Plateau, China. Field Crops Res. 2012;135:89-96. 